\title{
Validez ecológica y modelos de procesamiento de información
}

\section{Francisco Valle Arroyo}

\author{
Universidad de Oviedo
}

Si la finalidad última de la Psicología es explicar de manera cientifica la conducta humana, es decir, los procesos que tienen lugar en la vida de cada día tanto en las personas normales como en las anormales, todo el aparato experimental empleado debe ser tal que no modifique ni altere las condiciones que concurren en la experiencia cotidiana, sino que las mantenga y duplique en la medida de lo posible. Sin embargo, el afán de control experimental y el deseo de impedir la influencia de variables extrañas han producido, demasiado frecuentemente, situaciones experimentales completamente "asépticas» y por lo mismo totalmente diferentes de las cotidianas y presumiblemente los procesos mentales que tienen lugar en tales situaciones sean asimismo diferentes de los que se desarrollan en condiciones normales. Por lo mismo, las teorías o los modelos psicológicos propuestos, basados en los datos obtenidos en el laboratorio y que probablemente son debidos a la situación artificial del experimento, pudieran tener una validez circunscrita al entorno en que han sido elaborados, pero faltos de "validez ecológica». Este concepto en su sentido actual, no coincidente con el origina- rio de Brunswik (1956), hace referencia a la necesidad de equivalencia entre las condiciones experimentales y las de la vida real si se quiere que las teorías formuladas en base a los datos experimentales puedan aplicarse - servir de modelo- a las acciones o conductas que se dan en ambientes naturales, que son las que en último término se quieren explicar. Cuando tal equivalencia no se da, los resultados pueden ser irrelevantes para los fenómenos objeto de estudio.

Este planteamiento no es nuevo en absoluto, a pesar del boom literario del mismo, sino que de una forma $u$ otra ha sido una constante en la historia de la Psicologia. Ya en 1932, Bartlett critica acertadamente la posición de Ebbinghaus en los estudios de la memoria. Para éste, lo importante era lograr un control experimental riguroso y obtener la mínima variabilidad intersubjetiva, por lo que había que evitar a toda costa las experiencias y asociaciones previas del sujeto. La salida, históricamente genial, fue la utilización de sílabas sin sentido ya que éstas al no formar parte de la lengua no pueden verse afectadas por el aprendizaje anterior del sujeto; lo cual eviden- 


\section{Estudios}

temente no ocurre con las palabras. De este modo, pensaba Ebbinghaus, los datos obtenidos en los estudios experimentales podrian explicarse solamente por los mecanismos subyacentes -estructurales o procesuales - y no serían debidos a variables extrínsecas, experimentalmente incontrolables, como el pasado individual. Bartlett critica la postura de Ebbinghaus porque no se garantiza en absoluto esa pretendida uniformidad intersubjetiva ya que, utilizando sus propias palabras, los sujetos en un esfuerzo por encontrar sentido tratarian de hallarlo incluso en las sílabas desprovistas de significado, que al ser más indeterminadas harían que la variabilidad interindividual fuera aún mayor. (En cierto sentido, los supuestos de las técnicas proyectivas coinciden con el punto de vista de Bartlett: cuanto más impreciso o confuso es el estímulo presentado tanto más variable y subjetiva va a ser la respuesta dada.) Le critica también porque, al pretender estudiar la memoria "químicamente pura" se nos escapa de la mano nuestro objeto de estudio, a saber, la memoria humana, estudio en el que fundamentalmente se ha de dar cuenta y razón del por qué y del cómo retenemos y recuperamos material significativo. Como se puede ver, esta última crítica bartlettiana es la que realmente importa para la pregunta que nos hacemos en este artículo. ¿Hasta qué punto es lícito extrapolar los datos obtenidos en el laboratorio a situaciones más normales, más próximas al bic et nunc de cada situación?; ¿se pueden generalizar, sin más, los hallazgos de los estudios de la memoria, por ejemplo, a cómo aprendemos, retenemos y usamos la información adquirida en los intercambios sociales de cada día? De cualquier forma y a pesar de estas críticas, en una segunda lectura de Bartlett se podría pensar que, según él, la cuestión de la similitud o diferencia entre las tareas del laboratorio y las de la vida cotidiana no es demasiado importante ni decisiva en el sentido de que si el sujeto de alguna forma pone orden en lo desordenado, determinación en lo indeterminado y sentido en lo carente de él — «effort after meaning»- entonces aun los estudios experimentales, por muy artificiosos que fueran, y sus resultados tendrian una validez universal (al menos dentro de una cultura dada) porque los sujetos utilizarían en ellos las estrategias que son comunes en los procesos de memoria de cada día. El estudio de «memoriones" parece indicar que ese es el caso. Hunt y Love (1972) destacan la gran facilidad que VP tenía para desarrollar asociaciones significativas (proporcionar significado) a sílabas sin sentido, habilidad que se veía ayudada en este caso concreto por el conocimiento que VP poseía de lenguas diversas.

En una fecha más próxima y también dentro de una temática más cercana a la que aquí se trata - ya que a pesar del título nos limitaremos a los modelos de procesamiento de la negación-, Wason (1965), explicó la dificultad de las negativas en los experimentos, no por ninguna razón intrínseca a las mismas, como podía ser - y era normal aducir en aquella época bajo la influencia de Chomski (1957)el número de transformaciones optativas que el sujeto tenía que urilizar para pasar de la oración nuclear a la derivada (producción), o de ésta a la nuclear (comprensión), sino adoptando un punto de vista pragmático, es decir, suponiendo que tal vez la situación experimental no tiene en cuenta, no replica, las condiciones y circunstancias en las que se usan las negativas en la conversación. Con esta perspectiva diseñó un experimento en el que se distinguían dos tipos de negativas que él llamó objetivamente plausibles: aquellas que en el experimento desempeñan una de las funciones naturales de las negativas, a saber, el distinguir y contraponer la excepción a la norma, y objetivamente implausibles: las que violan este uso (para una aproximación a la distinción entre plausibilidad objetiva y subjetiva, véase Wason [1965] y Valle Arroyo [1979] para una elaboración más detallada). El tiempo de la negación plausible fue significativamente menor que el de la negación implausible. Estos resultados son realmente pertinentes. A simple vista pudiera pensarse que la tan aireada cues- 
tión de la validez ecológica podría resolverse no con un abandono de la experimentación y una vuelta a los métodos de observación más típicos de las ciencias sociales, como pretenden algunos -línea en la que tal vez el caso más significativo sea el de Neisser (1967, 1976, 1982), que ha pasado de ser un campeón de los métodos de la investigación del laboratorio y su significación para el estudio de la conducta en situaciones no artificiales a un cierto desprecio, se podría decir, de los estudios perfectamente controlados en el laboratorio en favor del estudio en contextos naturales- sino con una mayor verosimilitud y naturalidad en las condiciones y tareas experimentales.

Esto supuesto, vayamos un poco más en concreto al tema que se quiere des- arrollar: la validez ecológica de los modelos propuestos para explicar el procesamiento humano de la información contenida en oraciones negativas. Cuando en un experimento, un sujeto tiene que verificar una frase negativa, es decir, comprobar si es verdadera o falsa, su latencia es significativamente más alta que la de la frase afirmativa correspondiente; asimismo en la mayoría de los estudios realizados se ha encontrado una interacción entre el valor de verdad - verdadera (V), falsa $(F)$ - y el tipo sintáctico de la frase empleada -afirmativa (A), negativa (N)--, de forma que las AF son más difíciles que las $A V$, pero las NF son más fáciles que las NV (Clark, Carpenter y Just, 1973). Los tiempos de reacción (TR) estándares en este tipo de experimentos pueden verse en la figura 1.

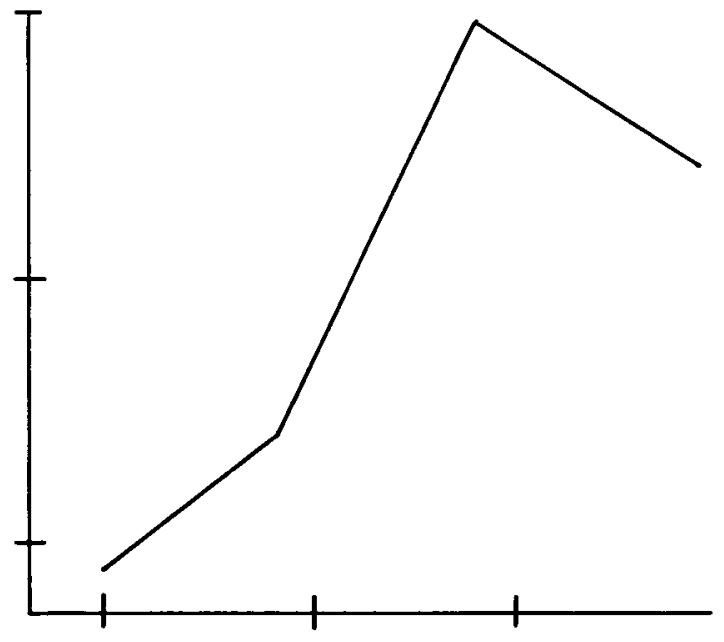

Fig. 1.-TR estandar.

Cualquier modelo de procesamiento de la negación ha procurado explicar estos dos hechos: mayor TR de las negativas e interacción entre sintaxis y semántica. Ahora bien: puediera ocurrir que ambos datos no fueran sino simples artefactos debidos a la situación experimental y que no tuvieran nada que ver con el modo en que procesamos las frases negativas en la vida real. Los modelos a los que se va a hacer referencia son los de
Clark (1976), Clark y Chase (1972), Trabasso (1970), Carpenter y Just (1975) y Greene (1972), si bien sólo el de Clark será expuesto con cierto detalle y se harán alusiones a los otros.

\section{EL MODELO DE H. CLARK}

Los supuestos teóricos y empíricos de su modelo pueden consultarse en Clark 
(1969, 1976), Clark y Chase (1972) y han sido expuestos con cierto detenimiento en castellano por Valero (1981). La tarea de los sujetos consistía en verificar una serie de enunciados del tipo «Star is (n't) above (below) plus» en relación con un dibujo que aparecía en la pantalla de una terminal de computadora y que consistía en un asterisco encima o debajo de un signo más $(+) \circ(;)$ (nótese la agramaticalidad de las frases usadas. Ambos sustantivos deben ir precedidos del artículo determinado. De hecho cuando el experimento se repitió utilizando frases más gramaticales «The star is below the plus", los resultados cambiaron sustancialmente, según comunicación personal de J. Gordon). El TR total empleado por el sujeto para responder $\mathrm{V}$ o F sería, según él, la suma de los tiempos parciales consumidos en cada una de las operaciones que tienen lugar. (Técnica de Sternberg, 1969.) Tales procesos eran los siguientes: 1. Codificación del dibujo; 2. Codificación de la frase; 3 . Comparación de ambas codificaciones, y 4 . Respuesta, pulsando el botón apropiado. Los estadios u operaciones 1,2 y 4 serían comunes a todos los tipos de frases y se podría decir que la suma de los tiempos de cada uno de dichos estadios constituiría el TR base ( $\left.t_{0}\right)$. Las diferencias en las latencias serian producidas por los distintos procesos involucrados en el estadio 3 . Cuando las codificaciones del dibujo y de la frase coincidían plenamente (AV), el TR coincidiría con el to. Por el contrario, a medida que el número de discordancias o diferencias entre ambas codificaciones era mayor, aumentaba el TR. Los componentes extra de latencia de los otros tres tipos de oraciones venían indicados por el parámetro $c$ en las $\mathrm{AF}$, los parámetros $(b+d)+c$ en las NV y los parámetros $(b+d)$ en las NF. O sea, los componentes de.latencia de cada uno de los tipos de frases serían éstos:

$$
\begin{aligned}
& \text { AV } \rightarrow t_{0} \\
& \text { AF } \rightarrow t_{0}+c \\
& N V \rightarrow t_{0}+b+d+c \\
& N F \rightarrow t_{0}+b+d
\end{aligned}
$$

Como se puede ver en el recuadro anterior, los TR obtenidos en los diferentes tipos de oraciones quedan suficientemente explicados (en general, el porcentaje de la varianza total explicado por el modelo supera con creces el 90 por 100) y en consecuencia los dos fenómenos a los que se aludía unas líneas más arriba. El único problema que queda por explicar es que el aumento del TR debería ser lineal, ya que los distintos parámetros miden el mismo tipo de operación - cambio del indicador del valor de verdad de un estado a otro-, presupuesto que han puesto de manifiesto empíricamente Carpenter y Just (1975) en su modelo de Comparación de Constituyentes.

En contraposición a las pautas de resultados obtenidos por Clark, en algunos estudios de Trabasso no se daba la interacción entre valor de verdad y forma sintáctica, sino que las oraciones falsas eran siempre más difíciles que las verdaderas fuera cual fuera su forma sintáctica. Clark habló, refiriéndose a los resultados de Trabasso, de modelo de "conversión» porque, según él, los resultados obtenidos se podrían explicar suponiendo que los sujetos transformaban las oraciones negativas en sus correspondientes afirmativas y una vez hecho esto el proceso de comparación se efectuaba como en su modelo. Lógicamente aquí aparecía un nuevo parámetro (k) que mediría el tiempo de traducción, común a todas las negativas; pero ahora en las NF habría además una discrepancia entre la codificación del dibujo y la de la frase, discrepancia que no se daba en las NV. El hecho de la posibilidad misma de esta traducción nos hace reflexionar sobre otra de las características de los atributos usados en la verificación de frases. En la mayoría de los estudios realizados sólo dos términos se han usado como predicados de las oraciones, por ejemplo, «encima/debajo», «dentro/fuera». La estrategia de los sujetos sería convertir «la estrella no está encima del signo más» en «la estrella está debajo del signo más». Este proceso, válido en sí siempre que se trate de conceptos binarios, y útil para 
resolver el problema podría manifestar por una parte, que tal como suponían estos modelos el sujeto tiene mayor dificultad en operar con conceptos negativos y por eso los transforma, pero al mismo tiempo, pone de manifiesto la artificialidad de la situación experimental, ya que en la vida normal no siempre actuamos con conceptos binarios. Por otra parte, el hecho experimental de preferir subjetivamente operar con oraciones afirmativas puede ser indicio de lo artificial de la situación en el sentido de que las negativas están fuera de contexto.

Expuesto someramente el modelo de Clark, se pasa a una descripción detallada del presente experimento. La idea era lograr una situación experimental en la que la función natural de las negativas no se viera alterada. En el lenguaje de cada día no usamos indiscriminadamente las negativas. Las afirmativas representan aproximadamente el 85 por 100 mientras que las negativas sólo ocupan un 8 por 100 del conjunto de frases del habla espontánea. Algunos (Goldman-Eisler y Cohen, 1970) han querido ver en esta diferencia de frecuencia de uso la explicación de la dificultad de las negativas, ya que las afirmativas constituirían un material sobreaprendido; posición que ha sido refutada por Wason y Johnson-Laird (1972) entre otros. Su uso es, por el contrario, selectivo, y uno de los usos de las negativas es, como ha afirmado Wason (1965), el contrastar y distinguir la excepción frente a la norma, es decir, que las negativas se usan para afirmar que algo o alguien no posee la cualidad que es compartida por los demás miembros del grupo. Así resultaría apropiado - plausible - el que un funcionario de cualquier oficina de (desempleo de una ciudad castellana dijera a otro:

1. Esta mañana se presentó a solicitar trabajo un hombre que no bablaba castellano, pero inadecuado, chocante y, en último término, inexpresivo:

2. Esta mañana se presentó a solicitar trabajo un hombre que no bablaba inglés (a no ser, claro está, que estuvieran refirién- dose a un puesto de trabajo para el cual el hablar inglés fuera un requisito exigido). La frase (1) sería plausible porque lo normal en Castilla es hablar castellano y en consecuencia el solicitante es una excepción de esa norma, mientras que (2) es inapropiada porque hace presuponer que lo corriente es hablar inglés cuando de hecho no lo es (véase Ayer [1952] y Givón [1978]).

En el experimento que aquí se describe se intentó crear una situación de plausibilidad y otra de implausibilidad (control). En el primer caso los sujetos antes de verificar una frase, de uno de los cuatro tipos tradicionales (AV, AF, NV, NF) tenían que decir - pulsando el botón apropiado- qué palabra de un conjunto de cuatro era distinta de las otras tres. En la situación implausible los sujetos no debían solucionar este problema, sino que directamente pasaban a la verificación de la frase. Así, ęn la primera situación se les podía mostrar a los sujetos este conjunto de palabras, cada una de ellas con un número de identificación debajo:

$$
\begin{array}{cccc}
\text { Brasil } & \text { Suecia } & \text { Alemania } & \text { Francia } \\
1 & 2 & 3 & 4
\end{array}
$$

Después de haber seleccionado «Brasil», pulsando la tecla 1 , por ser el único país americano frente a los demás auropeos, tenían que verificar una frase, referida a una de las palabras de la tétrada, que podía ser:

- AVS(emejante) = Suecia es un pais europeo.

- AFS(emejante) $=$ Suecia es un país americano.

- NVS(emejante) $=$ Suecia no es un país americano.

- NFS(emejante) = Suecia no es un país europeo.

- AVD(iferente) $=$ Brasil es un país americano.

- $\operatorname{AVD}$ (iferente) $=$ Brasil es un país americano.

- AFD(iferente) $=$ Brasil es un país europeo.

- NVD(iferente) $=$ Brasil no es un país europeo. 
- NFD(iferente) $=$ Brasil no es un país americano.

Como puede intuirse, las dos frases más relevantes para la idea que se estaba estudiando eran estas dos: "Suecia no es un país americano» y «Brasil no es un país europeo". La primera sería una negativa implausible, semejante a (2), mientras que la segunda tendría las caracteristicas de (1) -negativa plausible-, ya que negamos que la excepción (Brasil) tenga la cualidad que los otros miembros del conjunto comparten. Se esperaba que el tiempo de la negación plausible - NVD - fuera significativamente menor que el de la implausible - NVS—, pero sólo en el grupo plausible.

\section{METODO}

Diseño: Como ya se ha indicado había dos grupos experimentales a los que podemos llamar respectivamente: Grupo Plausible (GP) y Grupo Implausible (GI). El GP tenía que realizar dos tareas, una de selección del elemento dispar dentro de un conjunto y otra de verificación de una frase que se refería a uno de los elementos presentados en el problema de selección. El GI, por el contrario, sólo debía realizar la tarea de verificación. Cada sujeto, independientemente del grupo al que aleatoriamente hubiera sido asignado, tenía que verificar 64 oraciones, ocho de cada uno de los tipos antes descritos. El experimento tenía un diseño mixto con un factor inter y tres intra-grupo, cada uno de ellos con dos niveles. La variable intergrupo era Plausibilidad/Implausibilidad; los tres factores intra-grupo eran: Discrepancia/Semejanza, Afirmación/ Negación y Verdad/Falsedad. $\left(2^{3}=8\right.$ tipos de frases.)

Palabras y oraciones: Cada tétrada y cada oración era distinta de todas las demás. Sólo en el caso de que el sujeto cometiera algún error podía (aunque no necesariamente) repetirse alguna de las tareas de verificación. Cualquiera de las cuatro palabras que aparecían en el problema de selección podía funcionar como sujeto gramatical de la frase que el sujeto debía verificar. Todas las oraciones tenían la estructura sintáctica siguiente: « $S$ (no) es $\mathrm{P}$; donde $\mathrm{S}=$ sujeto y $\mathrm{P}=$ predicado. $\mathrm{El}$ predicado podía ser la cualidad común a los elementos semejantes de la tétrada (país europeo, por ej.), o aquella que distinguía el elemento excepcional (país americano) de los semejantes. Las categorías a las que pertenecían las palabras usadas en el problema de selección eran muy variadas: marcas de coches, árboles, flores, ciudades, países, pájaros, mamíferos, cadenas comerciales, etc.

Sujetos: Participaron 54 èstudiantes (chicos y chicas) del curso de Psicología General. Los datos de cuatro de ellos no fueron incluidos en el análisis estadístico final a causa de su gran número de errores en una o en las dos tareas. El análisis final, pues, está basado en los datos de 50 alumnos, 25 por grupo.

Materiales y procedimiento: Los estímulos se presentaban en la pantalla de una PDP-11, que informaba al sujeto de si su elección había sido correcta. Si el sujeto no acertaba en el problema de selección, la máquina le explicaba porqué su decisión había sido incorrecta y volvía a presentar la misma tétrada, aunque en orden diferente. Si el error se producía en el problema de verificación, la máquina anotaba el tipo de frase en el que se había producido y repetía, después de al menos ocho intentos, el mismo tipo de frase, aunque no necesariamente una frase idéntica. El número total de presentaciones, por tanto, era 64 más el número de errores cometidos por el sujeto. De este modo, al final del experimento se tenía el mismo número de observaciones correctas en cada tipo de oración.

\section{RESULTADOS}

La variable dependiente eran los TR. Sólo las latencias de las respuestas correctas fueron incluidas en los análisis estadísticos. Se comprobó, no obstante, que no había habido ningún tipo de compromiso 
entre velocidad de respuesta y precisión, en el sentido de que las respuestas más rápidas fueron, en general, las que menos errores produjeron y las equivocaciones se hacían más frecuentes a medida que los TR se alargaban.

TABLA 1

$T R$ medios (en meses) en los dos grupos experimentales

GP

\section{S}

\section{AV}

AF

NV

NF

NV - AV

\section{9}

2079

2406

2313

767
D

1624
1811
2036
2268
412

S

1918

2325

2729

2665

811
GI

\section{D}

1907

2206

2711

2559

804
TABLA 2

Número de errores por condición

\begin{tabular}{lrrrrr}
\hline & \multicolumn{3}{c}{ GP } & \multicolumn{3}{c}{ GI } \\
& S & D & S & D \\
AV & $\ldots \ldots \ldots \ldots \ldots \ldots \ldots \ldots \ldots$ & 5 & 5 & 16 & 16 \\
AF & $\ldots \ldots \ldots \ldots \ldots \ldots \ldots \ldots$ & 13 & 10 & 24 & 18 \\
NV & $\ldots \ldots \ldots \ldots \ldots \ldots \ldots \ldots$ & 20 & 5 & 31 & 21 \\
NF & $\ldots \ldots \ldots \ldots \ldots \ldots \ldots$ & 14 & 24 & 20 & 23 \\
\hline
\end{tabular}

En el GP los TR de las oraciones cuyo sujeto era una de las palabras semajantes - condición $\mathrm{S}$ - y los de las referidas al término discrepante -condición Dfueron bastante diferentes en las AF (2079 y 1811 mseg., respectivamente) y en las NV (2406 y 2036), y muy próximos en los otros dos tipos de oraciones -AV y NF-. Todos los efectos principales fueron significativos, como también estas dos interacciones: Negación $\times$ $\times \operatorname{Verdad}[\mathrm{F}(1,24)=8,6, p<\cdot 01]$ y Discrepancia $\times$ Negación $\times$ Verdad $[F(1,24)=14,38, p<.001]$. Tal y como se había supuesto las oraciones negativas NVD obtuvieron unos TR considerablemente más bajos (370 mseg.) que las NVS. Para ver si las NV plausibles eran significativamente diferentes de las implausibles se repitió el mismo análisis, pero sólo con las oraciones verdaderas. $\mathrm{Si}$, de hecho, lo eran, entonces la interac- ción Discrepancia $\times$ Negación sería significativa, y así ocurrió $[\mathrm{F}(1,24)=17,36$, $p<\cdot 001]$. Conviene notar también que los sujetos respondieron más rápidamente a las NVD que a las NFD, pero lo contrario ocurrió en la condición $\mathrm{S}$ del GP, y tanto en la condición $S$ como en la $\mathrm{D}$ en el GI. Estos resultados no pueden ser explicados ni por el modelo de Clark y Chase (1972), ni por el de Trabasso (1970), ni tampoco es válida la interpretación que da Greene (1972), como se verá con más detalle en la discusión.

En el GI no se esperaban diferencias sustanciales entre los TR de las oraciones referidas al término discrepante y los de las oraciones cuyo sujeto era una de las palabras semejantes, ya que no había que realizar la tarea de selección, considerada indispensable para que la plausibilidad produjera su efecto. Como puede comprobarse en la tabla 1, en todos los tipos de oraciones los TR fueron bastante semejantes. Merece particular atención el hecho de que en este grupo la diferencia entre las NVD y las NVS fue de 18 mseg., mientras que en el GP tal diferencia alcanzó los 370 mseg.

\section{DISCUSION}

Los resultados obtenidos en el GP y en el GI presentan diferencias radicales y que tienen que ver precisamente con los 
dos datos empíricos a los que se ha hecho referencia y que todos los modelos propuestos han tratado de explicar. Por una parte, el TR de la negación plausible, aun siendo todavía considerablemente superior al de las afirmativas correspondientes, descendió de un modo estadísticamente significativo (en un 50 por 100 aproximadamente) en relación con el tiempo de respuesta de las negativas implausibles. Por otra, la interacción entre sintaxis y semántica sólo se dio en la situación implausible (condición S del GP y condiciones S y D del GI). Teóricamente se podría pensar que las negativas son intrínsecamente difíciles y que en consecuencia los TR más altos obtenidos sistemáticamente en los experimentos reflejan sin más esta dificultad natural - punto de vista adoptado en general por los modelos de procesamiento de información negativa-, o se podría suponer que su dificultad se debe a causas extrínsecas, a la situación experimental que no habría sabido captar ni replicar la función natural de las negativas, como supuso Wason (1965) y como ha sido confirmado en este experimento. Mi impresión es que en la conversación de cada día las negativas no nos parecen más difíciles de entender que las afirmativas y que, por tanto, el uso indebido y artificial de las negativas pudiera ser el responsable de los TR extra empleados en su verificación experimental. Entonces, ¿por qué las negativas plausibles son todavía considerablemente más difíciles que las afirmativas? Tal vez nunca se pueda alcanzar en un laboratorio el contexto óptimo de un enunciado negativo, ya que en él es difícil no perder muchos de los factores lingüísticos, paralingüísticos y nolingüísticos que concurren en el intercambio comunicativo cotidiano y que forman parte del contexto total (SlamaCazacu, 1970) facilitando la comprensión. Pero si se toman las debidas precauciones para aproximarse experimentalmente a estas condiciones óptimas, entonces las negativas no deberían ser más difíciles de comprender que las afirmativas, como parece que ocurre en la conversación y como ha puesto de mani- fiesto experimentalmente Gordon (1978) $\mathrm{y}$, tal vez, también De Villiers \& TagerFlusberg (1975), dada la pequeña diferencia entre los TR de las afirmativas y negativas en su grupo de edad más avanzada.

De este estudio se puede sacar una conclusión importante. El conjunto de datos obtenidos pudiera considerarse como un test acerca de la validez ecológica de los modelos de procesamiento de la negación y, al mismo tiempo, una refutación de algunas de las explicaciones que se han dado de porqué las negativas obtienen respuestas más lentas en los experimentos. Estos modelos de procesamiento (Clark y Chase, 1972; Trabasso, Rollins y Shaugnessy, 1971; Carpenter y Just, 1975) pueden ser excelentes cuando tratan de predecir las latencias que los distintos tipos de enunciados obtienen en los experimentos -después de todo eso es el fin con el que fueron desarrollados-, y sin duda reflejan las diferentes estrategias que los sujetos pueden usar para habérselas con oraciones fuera de contexto o con un contexto limitado y parcial; pero ¿hay algún parecido entre estas tareas y la comprensión de los enunciados negativos dados en la conversación? ¿Cómo podrían explicar estos modelos las pautas de latencias, radicalmente diferentes, producidas en las situaciones plausible e implausible? ¿Por qué habían de ser -desde su punto de vista- las NVD mucho más rápidas que las NVS si los componentes de latencia son exactamente los mismos? Sin embargo, si se adopta el punto de vista funcional y pragmático, defendido en este artículo, la explicación resulta bastante fácil, ya que éstas son las únicas condiciones en las que las negativas cumplen una función semejante a la que desempeñan en el lenguaje natural. Del mismo modo, dados los resultados de este estudio, la interacción entre negación y valor de verdad no se explica suficientemente ni en el modelo de «verdad» de Clark (1976), ni en el de "conversión", de Trabasso (1970). El primero podría explicar las latencias de la situación implau- 
sible, puesto que los resultados en esta condición son semejantes a los suyos, pero ¿cómo se puede explicar con su modelo el que en el mismo experimento, con los mismos sujetos y con un procedimiento idéntico, las NV sean más fáciles que las NF en la situación plausible? En una posición, aún más complicada, se encuentra el modelo de Trabasso (1970). Este modelo podría explicar el orden creciente de los TR de la situación plausible y no sería capaz de dar cuenta de los de la situación implausible, pero su modelo se apoya básicamente en el supuesto de que los sujetos traducen las oraciones negativas a las afirmativas equivalentes. Ahora bien, en el presente estudio no puede hacerse esta traducción, ya que, en primer lugar, el experimento no trataba de conceptos binarios en los que son razonables tales conversiones y, además, el sujeto no sabía de antemano la forma exacta que iban a tener las frases, por lo que las traducciones habrían sido inútiles e imposibles. Aunque el punto de vista de Greene $(1970,1972)$ es mucho más cercano al defendido en este artículo, su explicación de la inversión de los TR de las NV y NF me parece insostenible. Ella supone que usamos las negativas para señalar un cambio de significado, postura no sustancialmente diferente de $\mathrm{mi}$ interpretación, pero argüir que las negativas falsas (NF) son más fáciles, es decir, tienen TR menores, porque cumplen su función natural - cambio de significado- al "negar la situación presentada en el dibujo» mientras que las NV se usan para desempeñar «el papel no natural de afirmar lo que se da en el dibujo sin cambiar el significado» (1972, pág. 121) no puede sostenerse; y esto por dos razones. Primera, porque, como en los dos modelos anteriores, la explicación dada es válida sólo para un grupo de datos (la condición implausible) y no para la plausible; y, segunda, porque de acuerdo con su razonamiento, las NF serían las negativas plausibles, lo cual es claramente incorrecto. Un enunciado falso no puede ser nunca un enunciado plausible (a no ser en circunstancias muy especiales, como puede ser un juicio, pongo por caso), ya que el lenguaje tiene como función primordial informar a nuestros interlocutores, no desinformarlos o engañarlos.

En el presente estudio se han obtenido dos pautas de respuestas (TR) completamente diferentes: la de la situación plausible y la de la implausible. La mayoría de los estudios realizados sobre la negación no han tenido en cuenta esta distinción entre plausibilidad $e$ implausibilidad y han propuesto tareas que, desde $\mathrm{mi}$ punto de vista, pudieran considerarse como implausibles - los datos así lo confirman-. Ahora bien, como los modelos de procesamiento de la negación se han elaborado en base a datos de este tipo y han explicitado los procesos que producirían los TR obtenidos en los experimentos y sus componentes de latencia, cualquier intento de aplicar tales modelos más allá de los límites del laboratorio, por ejemplo, a la comprensión de las negativas en la vida real puede verse inexorablemente invalidado, ya que las pautas de respuesta obtenidas en la situación plausible -más próxima a la real- son radicalmente distintas; por lo cual se hace necesario construir modelos, apoyados en estos datos, que tendrán naturalmente mayor validez ecológica. (Un modelo de negación plausible ha sido desarrollado en otra parte, Valle Arroyo, 1979.)

Otra alternativa posible es limitar la aplicabilidad de los modelos al ambiente y situación específicas en que fueron elaborados, aunque esto plantea problemas de otra indole que no es el momento de comentar. [Muy diferente es, por ej., publicar un artículo con el título «On the process of comparing sentences against pictures» (Clark \& Chase, 1972) a escribir un libro con el de «Semantics and comprehension» (Clark, 1976)].

Y una nota final. Este artículo ha sido escrito aceptando en principio el punto de vista de los defensores de la validez ecológica y, por lo mismo, sujeto a las mismas críticas que aquéllos, entre las que se pueden citar, por ej., la falta de concreción de qué se entiende por natu- 
ral y artificial, la tal vez artificiosa, o al menos inespecificada, distinción entre experimentación y observación, y la difícil demarcación entre una y otra, que, en cierto modo, sería una reformulación de la distinción entre Naturaleza y Cultura, que tampoco es tan clara ni tan fácil de precisar. (Agradezco a Tomás R. Fernández por haberme señalado con lucidez y precisión este último punto.)

\section{Referencias}

AYER, A. J.: “Negation». The Journal of Philosopby, 1952, 49, 797-15.

BARtlett, F. C.: Remembering. A Study in Experimental and Social Psychology. Cambridge: C. U. P., 1932.

Brunswik, E.: Perception and the Representative Design of Psychology. Berkeley: Univ. Cal. Press, 1956.

CARPENTER, P. A., y JUST, M. A.: "Sentence comprehension: a psycholinguistic processing model of verification». Psychological Review, 1975, 82, 45-73.

Clark, H. H.: "Linguistic processes in deductive reasoning". Psychological Review, 1969, 76, $387-404$.

ClaRK, H. H.: Semantics and Comprehension. La Haya: Mouton, 1976.

Clark, H. H.; CARPEnTER, P. A., y Just, M. A.: "On the meeting of semantics and perception", en W. G. Chase (ed.), Visual Information Processing. N. York: Academic Press, 1973.

Clark, H. H., y CHASE, W. G.: «On the process of comparing sentences against pictures». Cognitive Psychology, 1972, 3, 472-517.

Chomsky, N.: Syntactic Strutures. La Haya: Mouton, 1957. (Trad. cast., Estructuras sintacticas, Siglo XXI, 1974.)

De Villiers, J. G., y Tager-Flusberg, H. B.: «Some facts one simply cannot deny". Journal of Child Language, $1975,2,279-286$.

Gıvón, T.: "Negation in language: pragmatics, function, ontology", en P. Cole (ed.), Syntax and Semantics. Vol. 9, Pragmatics. N. York: Academic Press, 1978.

Goldman-Eisler, F., y COHEN, M.: «Is N, P, and PN difficulty a valid criterion for transformational operations?" Journal of Verbal Learning and Verbal Bebavior, 1970, 9, 161-166.

GORDON, J. F.: «The presuppositions underlying plausible negation». Artículo leido en el Congreso de la APA, 1978.

GREENE, J. M.: «The semantic function of negatives and passives». British Journal of Psychology, 1970, 61, 17-22.

Greene, J. M.: Psycholinguistics. Chomsky and Psychology. Suffolik: The Chaucer Press, 1972. (Trad. cast., Psicolingüistica. Chomsky y la Psicologia, Trillas, 1980).

Hunt, E., y Love, T.: "How good can memory be?", en A. W. Melton y E. Martin (ed.), Coding Processes in Human Memory. Washington: Winston, 1972.

NeIsSer, U.: Cognitive Psychology. N. York: Appleton, 1967. (Trad. cast., Psicología Cognoscitiva, Trillas, 1976.)

NeISSER, U.: Cognition and Reality. San Francisco: Freeman, 1976. (Trad. cast., Procesos Cognoscitivos y Realidad. Marova, 81.)

NeISSER, U.: Memory Observed. Remembering in Natural Contexts. N. York: Freeman, 1982.

SLAma-CazACU, T.: Lenguaje y Contexto. Barcelona: Grijalbo, 1970.

STERNBERG, S.: «Memory-scanning: mental processes revealed by reaction-time experiments». American Scientist, $1969,57,421-57$.

TrabASSO, T.: «Reasoning and the processing of negative information». Conferencia inaugural del 78 Congreso de la APA, 1970.

Trabasso, T.; Rollins, H., y Shaugnessy, E.: «Storage and verification stages in processing concepts». Cognitive Psychology, 1971, 2, 239-289.

VALERo Burguete, E.: «El procesamiento cognitivo de las oraciones negativas». Estudios de Psicología, $1981,5 / 6,201-214$

VALLe ARroYo, F.: Negation in Objetively Plausible Contexts. University Microfilms International, n. $79-21840$.

WASON, P. C.: "The contexts of plausible denial".Journal of Verbal Learning and Verbal Bebavior, 1965, 4, 7-11.

Wason, P. C., y Johnson-LaIRD, P. N.: «Psychology of Reasoning». Structure and Content. Londres: Batsford, 1972. (Trad. cast., Psicología del Razonamiento. Debata, 1980.) 


\section{Resumen}

Dos de los ballazgos más frecuentemente repetidos en la intestigación sobre el procesamiento de las negaticas: mayor $T R$ de éstas que de las afirmativas correspondientes $e$ interacción entre sintaxis y semántica son considerados en este estudio como posibles artefactos de la situación experimental, que no ba sabido, en general, tener en cuenta la función de las negativas en la conversación. Cuando en una situación de laboratorio se procura reproducir tal función, el tiempo de la negación disminuye de manera estadísticamente significativa y además no se da la interacción a la que se alude más arriba. Basándose en estos resultados se aboga por una mayor plausibilidad experimental que puede incrementar la validez ecológica de los modelos de procesamiento.

\section{Abstract}

Two of the most frequently found experimental facts in information processing research on negatives - RT of negatives higher than that of affirmatives and interaction between syntax and semantics - are considered as possible artifacts due to the experimental situation that, in general, does not take into account the function negatives play in everyday contersation. When in a laboratory situation one tries to recreate this function, negation time decreases in a statistically significant way and, moreover, the interaction cited above does not show up. Based on these results a better experimental plausibility, that may increase the ecological validity of information processing models, is defended.

\section{Résumé}

Les deux faits les plus frequemment trouvés dans la recherche scientifique sur le processus des négativies - un TR plus long pour les negatives que pour les affirmatives et une interaction entre syntaxe et semántique- sont considerés ici comme de pures artifices de la situation expérimentale, qui, en general, $n$ ' a pas su tenir compte de la fonction des négatives dans la contersation. Quand dans une situation de laboratoire on essaie de reproduire telle fonction, le temps de négation diminue de manière statistiquement significative et, en plus, ne se troute pas l'interaction citée ci-dessus. D'après ces resultats, on defend une meilleure plausibilité expérimentale qui pourrait accrồtre la validité écologique des modeles de processus. 